\title{
Technè
}

La science au service de l'histoire de l'art et de la préservation des biens culturels

46 | 2018

Science et conservation

\section{La protection cathodique par anodes sacrificielles : adaptation du système pour la Sphinge " au parapluie ", épreuve en béton armé}

Sacrificial anode cathodic protection: adapting the system for the Sphinge "au parapluie", a sculpture in reinforced concrete

\section{Claire Brière}

\section{OpenEdition}

Journals

Édition électronique

URL : https://journals.openedition.org/techne/483

DOI : 10.4000/techne.483

ISSN : 2534-5168

Éditeur

C2RMF

Édition imprimée

Date de publication : 1 décembre 2018

Pagination : 104-108

ISBN : 978-2-11-152829-1

ISSN : 1254-7867

\section{Référence électronique}

Claire Brière, "La protection cathodique par anodes sacrificielles : adaptation du système pour la Sphinge " au parapluie », épreuve en béton armé », Technè [En ligne], 46 | 2018, mis en ligne le 19 décembre 2019, consulté le 21 septembre 2021. URL : http://journals.openedition.org/techne/483 ; DOI : https://doi.org/10.4000/techne.483

\section{cc) (†)}

La revue Technè. La science au service de l'histoire de l'art et de la préservation des biens culturels est mise à disposition selon les termes de la Licence Creative Commons Attribution - Pas d'Utilisation Commerciale - Pas de Modification 4.0 International. 


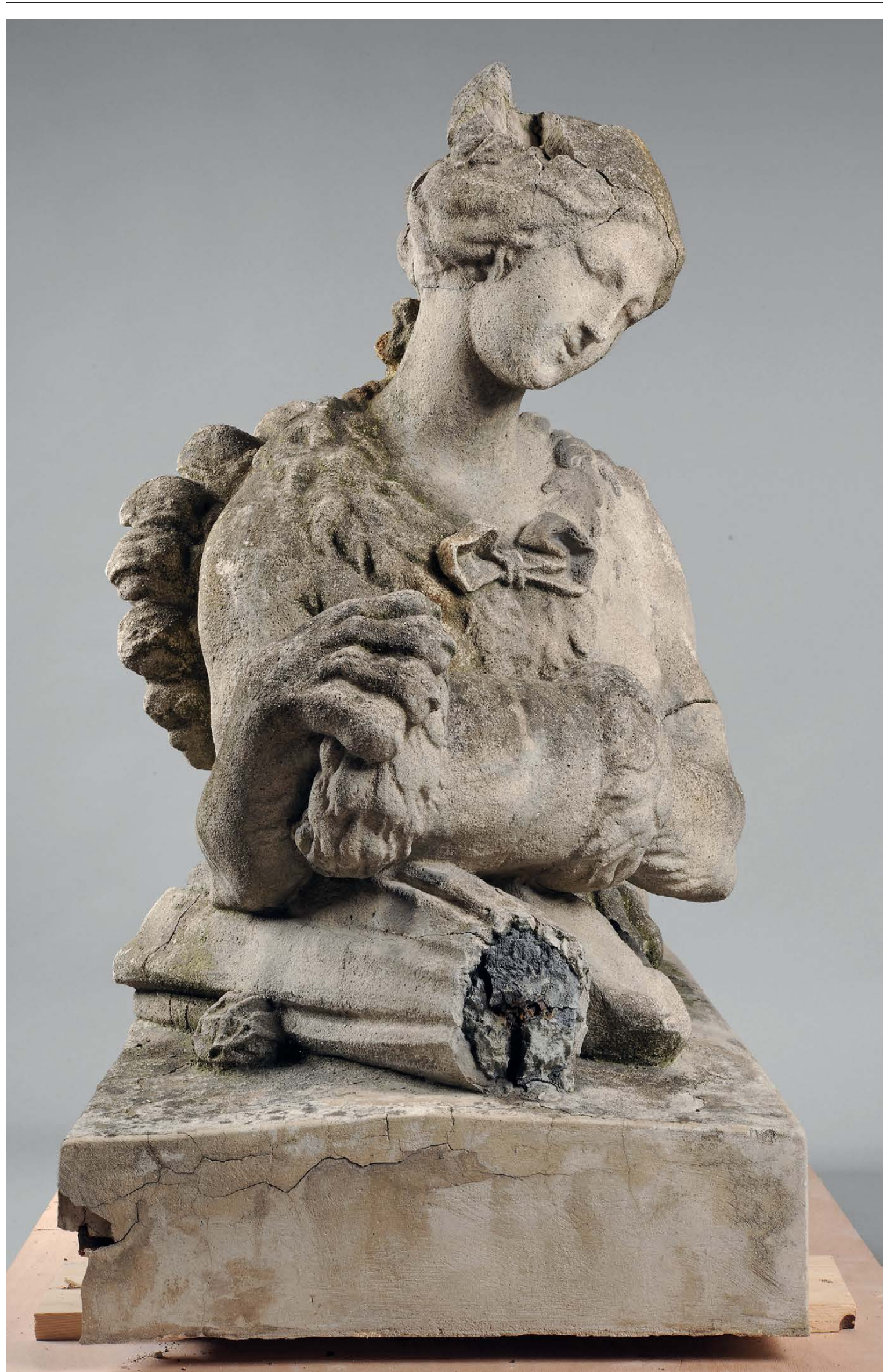

Fig. 1. Anonyme, Sphinge " au parapluie », stylistiquement datée des années 1900, épreuve en béton armé moulé, de face avant restauration (105 x $125 \times 59 \mathrm{~cm})$. ( Inp/G. Vanneste. 


\section{La protection cathodique par anodes sacrificielles : adaptation du système pour la Sphinge "au parapluie », épreuve en béton armé}

Sacrificial anode cathodic protection: adapting the system for the Sphinge "au parapluie", a sculpture in reinforced concrete

Résumé. La protection cathodique par anodes sacrificielles est un procédé de stabilisation des armatures corrodées dans un béton. Il s'agit d'un traitement électrochimique qui permet de réduire considérablement la cinétique de corrosion des armatures. Le procédé est largement exploité dans le secteur des bâtiments et des travaux publics. Il est, depuis une dizaine d'années, également utilisé pour la restauration des monuments historiques en béton. La restauration de la Sphinge « au parapluie », sujet de notre mémoire à l'Institut national du patrimoine, fut l'occasion d'étudier l'adaptabilité du traitement à une petite échelle. Cet article présente la synthèse de notre travail.

Mots-clés. Protection cathodique, corrosion, galvanique, anode, armature, béton, oxydation.

\begin{abstract}
Sacrificial anode cathodic protection (SACP) is a technique used to control metal corrosion in reinforced concrete. It is an electrochemical process that considerably reduces the kinetics of corrosion of reinforcing steel bars. The process is widely used in civil engineering. For the past ten years or so, it has also been used in the restoration of historical monuments made of concrete. The restoration of the Sphinge "au parapluie" (Sphinx "with an umbrella"), the subject of my thesis at the Institut national du patrimoine, Paris, provided an opportunity to study the application of this technique on a small scale. This paper presents an overview of the work undertaken.

Keywords. Cathodic protection, corrosion, galvanic, anode, reinforced concrete, oxidation.
\end{abstract}

Les sculptures en béton armé se dégradent inévitablement avec le temps. La corrosion de leurs armatures ferreuses provoque leur fissuration, leur fracturation jusqu'à parfois leur ruine totale. Il est donc impératif de stopper le processus de corrosion ou, tout du moins, de le ralentir au maximum. La protection cathodique avec anodes sacrificielles stabilise les armatures d'un béton en utilisant le principe de la corrosion galvanique. Ainsi, lorsque les méthodes traditionnelles de lutte contre la corrosion dans le domaine patrimonial (revêtements : vernis et cire, inhibiteurs, convertisseurs de rouille) ne peuvent pas être utilisées, elle peut être une bonne alternative.

Ce procédé a fait ses preuves dans le bâtiment : il est efficace. Installées préventivement, les anodes sont implantées dès la construction de la structure. Dans le domaine des Monuments historiques, l'emploi curatif des anodes semble bien fonctionner également, malgré quelques difficultés (détection précise des armatures, remplacement des anodes, contrôle d'efficacité, etc.). Si le système n'est pas encore utilisé pour la restauration des sculptures, c'est parce qu'il n'est pas compatible, à l'heure actuelle, avec les ouvrages de petit et moyen format. Il est très intrusif, nécessitant de multiples percements, et soulève ainsi des questions techniques et déontologiques. Le procédé doit donc être adapté.

\section{La Sphinge « au parapluie »}

La Sphinge " au parapluie » fut le sujet de notre mémoire de fin d'étude à l'Institut national du patrimoine (Inp) en $2015^{1}$ (fig. 1). Il s'agissait d'une épreuve en béton de chaux armé dont le modèle était daté des années 1900. La sculpture (105 x $125 \times 59 \mathrm{~cm}$ ) était creuse, ses armatures ferreuses ayant été placées entre les couches de béton lors du procédé de moulage. Le béton était composé d'une chaux hydraulique, de sable et de fragments d'une roche gris-rose ${ }^{2}$.

Jusqu'en 2015, la Sphinge était exposée dans la cour de l'Hôtel de Lamarck, résidence de l'ambassadeur de Belgique à Paris. Conservée en extérieur, la sculpture présentait de nombreuses altérations : d'épaisses croûtes noires, une forte colonisation par les micro-organismes, une érosion généralisée avec perte totale de l'épiderme, des colorations dues à des oxydes métalliques, etc. La corrosion de ses armatures avait provoqué la fissuration et l'éclatement du béton en divers endroits.

Au préalable des problématiques de nettoyage et de consolidation structurelle, le traitement des fers apparut comme une priorité. Sans ce dernier, aucune intervention de restauration (comblement, collage) n'aurait pu être pérenne. Cependant, les armatures enrobées ne pouvaient pas être 
isolées avec un revêtement de surface, ni traitées avec des inhibiteurs de corrosion du fait d'une forte présence de sulfates. Le convertisseur de rouille acide tannique ne pouvait pas non plus être utilisé du fait de son $\mathrm{pH}$ trop bas pour la chaux.

Ainsi la Sphinge apparaissait, du fait de ses altérations caractéristiques et de la problématique du traitement de ses armatures, assez représentative de nombreuses sculptures exposées aujourd'hui dans les espaces publics. Pour ces œuvres, la protection cathodique est une réelle piste à explorer.

La Sphinge fut ainsi le support de cette étude, menée conjointement avec Élisabeth Marie-Victoire et Myriam Bouichou, ingénieures au Laboratoire de Recherche des Monuments Historiques, et Christian Tourneur, directeur scientifique et technique de l'entreprise Freyssinet ${ }^{\circledR 3}$. Ce dernier fournit le matériel nécessaire (anodes sacrificielles Foreva ${ }^{\circledR}$ Galvastar et équipement électrique) et résuma ainsi les enjeux dans son rapport A10-004R1-REF 001, Sphinge INP-Développement-Adaptation Galvastar-Rapport d'essais :

«Il s'agit d'une structure en béton armé délicate et dont les dimensions ne sont pas en accord avec les produits usuellement utilisés [...]. L'objectif d'étude est d'adapter les techniques de mises en œuvre et les produits standards Freyssinet aux dimensions de l'œuvre. »

\section{La protection cathodique avec anodes sacrificielles : principe et utilisation}

La corrosion galvanique fait intervenir deux métaux au potentiel de corrosion E différent ${ }^{4}$. Dans le cas de la protection cathodique, le couple est constitué du fer (acier des armatures) et du zinc (métal d'apport), avec EZn $<$ EFe.

Lorsque le fer et le zinc sont plongés dans un même électrolyte ${ }^{5}$, les réactions propres à la corrosion de chaque métal se déroulent à leurs surfaces :

- Oxydation du métal à l'anode $: \mathrm{Fe} \rightarrow \mathrm{Fe}^{2+}+2 \mathrm{e}^{-}$et $\mathrm{Zn} \rightarrow$ $\mathrm{Zn}^{2+}+2 \mathrm{e}^{-}$(réactions simplifiées);

- Réduction des espèces oxydantes à la cathode : $\mathrm{O}_{2}+{ }_{2} \mathrm{H}$ $\mathrm{O}+4 \mathrm{e}^{-} \rightarrow 4 \mathrm{OH}^{-}$(exemple de réaction en milieu alcalin) ;

- Combinaison des espèces formées et précipitation des produits de corrosion.

Lorsque les deux métaux sont également reliés électriquement, l'équilibre des réactions est bouleversé. Puisque le zinc possède le potentiel de corrosion le plus faible, cela signifie que son oxydation libère davantage d'ions et d'électrons dans le milieu. Les électrons produits par la dissolution du zinc vont ainsi se déplacer naturellement dans le circuit jusqu'au fer, pour maintenir l'équilibre électrique. Cette déportation déplace l'équilibre des réactions d'oxydo-réduction : le zinc s'oxyde toujours davantage tandis que le fer devient essentiellement le siège des réactions de réduction (fig. 2). Si l'on accepte la dégradation du zinc, le fer se trouve ainsi protégé.
Sur ce principe, la protection cathodique crée volontairement la circulation d'un courant électrique entre les armatures d'un béton et des anodes en zinc ${ }^{6}$. Celles-ci sont dites sacrificielles car, destinées à se corroder «à la place " des armatures, elles s'usent. Ainsi, elles ont une durée de vie limitée tributaire de leur taille, de la vitesse de leur consommation et de nombreux autres critères. Totalement oxydées, elles doivent être changées.

\section{Stratégie de protection}

Pour une structure, quelle qu'elle soit, le « dimensionnement » de l'installation constitue la première étape. Il s'agit d'évaluer les besoins en courant des armatures à préserver en fonction de la corrosivité du milieu et de l'environnement, et ainsi de déterminer le nombre d'anodes nécessaires, leur taille, leur position, la distance de l'une à l'autre, etc. Cette étape doit être réalisée par un bureau d'étude agréé (norme NF EN ISO 12696). Pour la Sphinge, le dimensionnement fut effectué par Christian Tourneur.

Dans un premier temps, plusieurs radiographies de l'œuvre furent prises à l'Inp par G. Vanneste afin de connaître précisément le nombre et la répartition de ses armatures (fig. 3). Une vingtaine de fers fut révélée, de trois types : ronds et fins $(\varnothing: 0,7 \mathrm{~cm})$ dans le corps de la Sphinge, à section en $\mathrm{T}(1: 2,5 \mathrm{~cm})$ dans sa tête et sa base, et des fers plats ( 1 : $2,8 \mathrm{~cm}$ ) dans la base également. Les armatures n'étaient pas reliées entre elles.

Pour une protection optimale de ce type de structure, les anodes devaient être ponctuelles, c'est-à-dire placées une par une au contact des fers. La sculpture devait être percée, l'anode insérée dans le béton, soudée à l'armature et recouverte d'un mortier poreux assurant le contact électrolytique.

Le rayon d'action des anodes fut estimé à $10 \mathrm{~cm}$ : les Galvastar ${ }^{\circledR}$ devaient être implantées tous les $20 \mathrm{~cm}$ le long de chaque fer. Le grand nombre de percements induit était problématique : au-delà de l'altération esthétique indéniable,

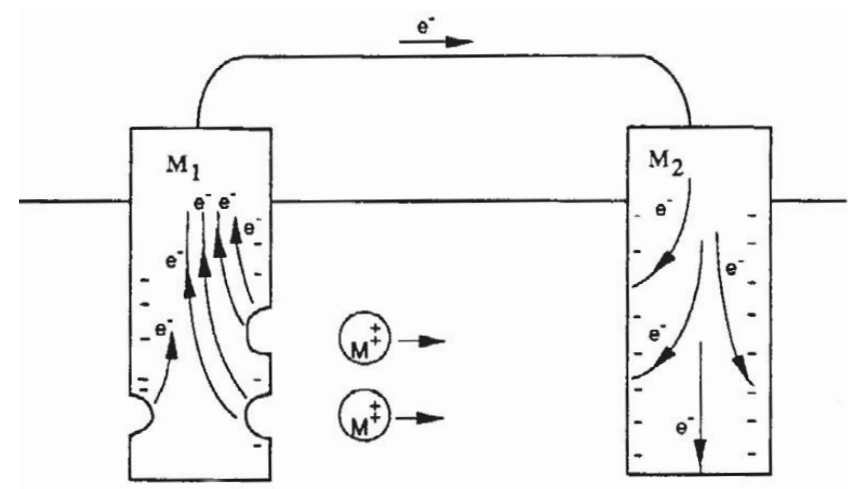

Fig. 2. Couplage galvanique : le zinc (M1) et le fer (M2) sont plongés dans un même électrolyte. ( ) J.-C. Marec. 


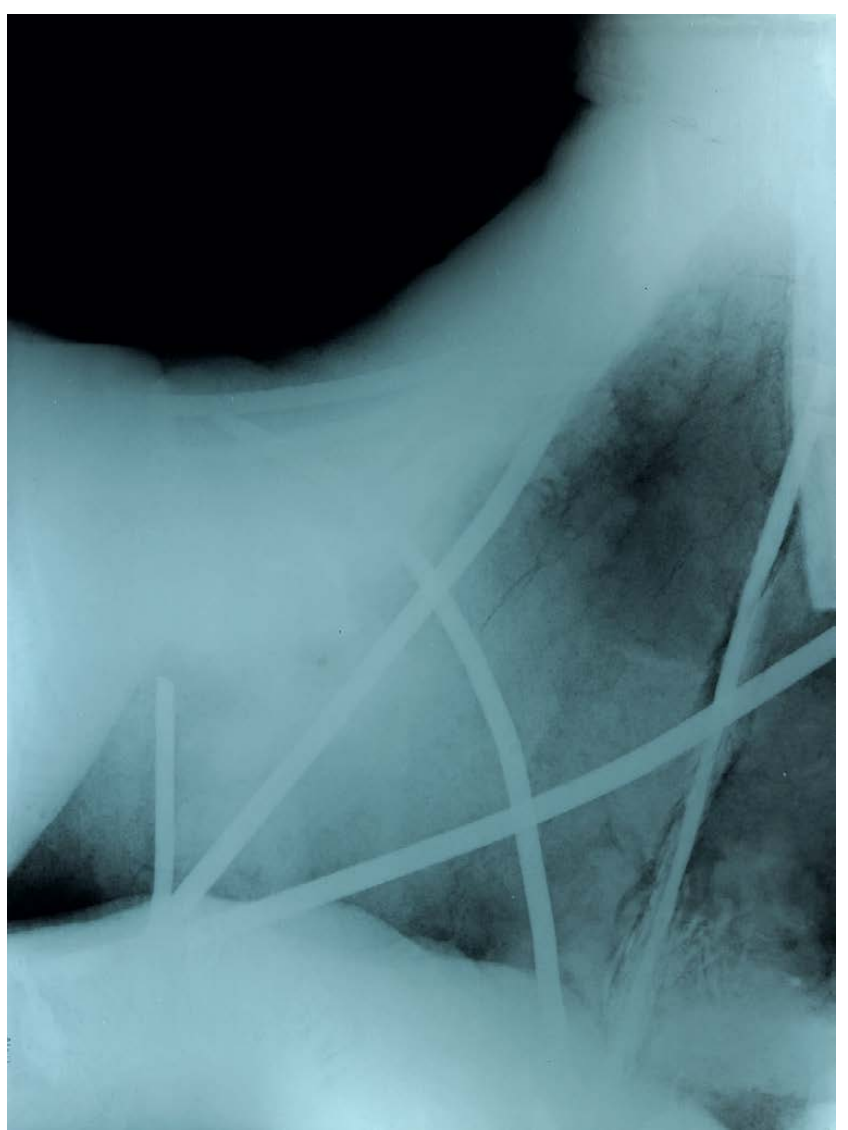

il aurait fragilisé incontestablement la structure. Il fut donc décidé de protéger uniquement les armatures provoquant les plus grands désordres structurels. Pour ce faire, nos relevés d'altérations ainsi que les radiographies, où les sections oxydées et feuilletées du métal se distinguaient parfaitement des sections saines, ont été utilisés. Selon ce compromis, seules les armatures du socle, de la tête et du corset côté dextre devaient recevoir des anodes (25 au total - fig. 4).

\section{Percement de l'enrobage béton}

Une fois le dimensionnement terminé et la stratégie de protection décidée, des essais de percement de béton furent entrepris ${ }^{7}$. En effet, l'insertion des anodes sacrificielles est un travail délicat et là où des percements peuvent être discernables mais discrets sur la façade d'un édifice, ils peuvent être ostensibles sur une sculpture.

Nous avons ainsi choisi de carotter plutôt que de percer. Pour cela, un foret creux diamanté de $25 \mathrm{~mm}$ de diamètre externe a été utilisé. Le foret entamant le béton sur $3 \mathrm{~mm}$, il permettait l'obtention d'une carotte nette pouvant, une fois coupée, être replacée dans la cavité après insertion de l'anode, tel un bouchon. Ainsi, en surface, seul un cercle

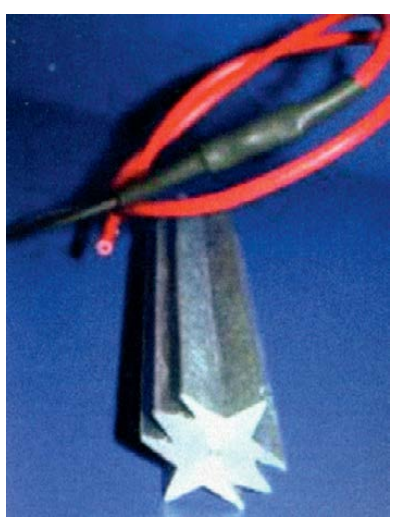

Fig. 3. Radiographie du corset. Une section d'armature particulièrement corrodée est visible en partie basse : la silhouette de la barre apparaît moins nettement, le métal semble "émietté ». () Inp/G. Vanneste.

Fig. 5. Anode Foreva ${ }^{\circledR}$ Galvastar. $\mathrm{L}=8 \mathrm{~cm}$. (C) Freyssinet.

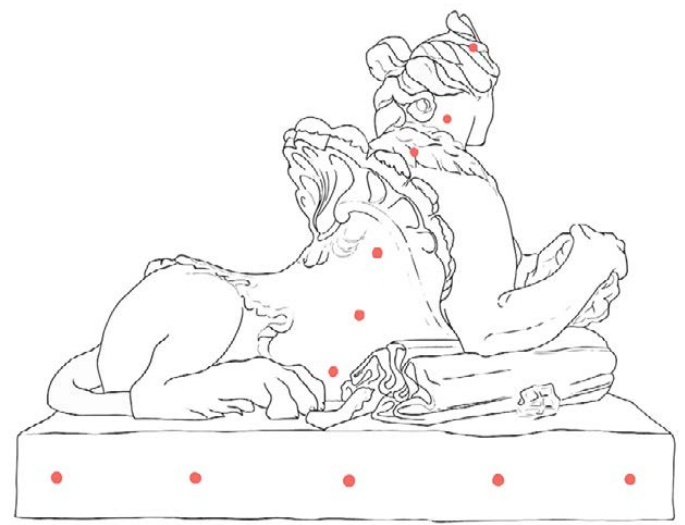

Fig. 4. Schéma d'implantation des anodes côté dextre. (c) C. Brière.

apparaissait, et non un disque. Le comblement était beaucoup plus fin, l'impact esthétique moindre.

Les essais permirent en outre de trouver les bons gestes. La complexité majeure résidait dans l'entaille de l'armature par le foret : en effet, l'acier diamanté sciait le métal aussi bien que le béton. Si la profondeur de l'armature n'était pas connue, celle-ci risquait d'être simplement coupée. Si le bruit émis par le foret pouvait être un bon indicateur en annonçant l'attaque du métal, nous préférâmes agir en deux temps : réalisation d'une carotte de l'enrobage avec le foret creux, puis dégagement de l'armature avec de petits ciseaux.

Enfin, les essais révélèrent la nécessité absolue d'utiliser un guide d'amorçage pour éviter toute éraflure en surface et permirent d'évaluer les vibrations et les risques encourus par la structure de l'œuvre.

\section{Adaptation du matériel}

Parallèlement aux essais de percement, le matériel fut transformé. Les anodes Foreva ${ }^{\circledR}$ Galvastar, commercialisées par le groupe Freyssinet, furent choisies pour la protection cathodique de la Sphinge. Leur morphologie n'étant pas adaptée ${ }^{8}$, 
longueur et connexion électrique notamment, elles furent modifiées (fig. 5).

Les Galvastar ${ }^{\circledR}$ étant trop longues pour être insérées dans la paroi de la Sphinge dont l'épaisseur n'excédait pas 2,5 cm par endroits ${ }^{9}$, elles furent donc coupées par l'équipe Freyssinet, réduites de 8 à $2 \mathrm{~cm}$, de 80 à $20 \mathrm{~g}$.

De plus, les Galvastar ${ }^{\circledR}$ ne sont pas des anodes ponctuelles : un fil de titane assure la connexion entre l'anode et un fil électrique général, lui-même soudé à l'armature à protéger. Ce schéma correspond aux anodes agencées «en chapelet ", système utilisé pour les bâtiments et travaux publics, inenvisageable pour la Sphinge. Par conséquent, Christian Tourneur et son équipe conçurent un nouveau schéma de connexion pour des Galvastar ${ }^{\circledR}$ unitaires. Le fil de titane fut supprimé et remplacé par une tige filetée en fer. Puis une méthode de soudure de la tige sur l'armature fut développée. Une fois la tige soudée, l'anode pouvait y être vissée directement dans la cavité du béton et recouverte d'un mortier assurant le contact électrolytique (passage des ions). Des tests de continuité électrique furent pratiqués afin de vérifier la viabilité du nouveau système : tous furent positifs.

À la suite de la modification morphologique des anodes, le dimensionnement du système fut repris. Si la sculpture était replacée dans la cour de l'Hôtel de Lamarck, la durée de vie des Galvastar ${ }^{\circledR}$ était estimée à 99 ans.

\section{Conclusion}

À l'issue de l'étude, les anodes sacrificielles ne furent pas insérées dans la Sphinge " au parapluie » car l'œuvre fut finalement exposée dans le hall de l'hôtel après restauration. Plusieurs raisons argumentèrent cette décision. Si les armatures de la sculpture étaient stabilisées grâce à la protection cathodique, une nouvelle exposition en extérieur signifiait la poursuite de la dissolution du béton et la perte progressive des volumes, ainsi qu'une nouvelle colonisation biologique. Installer l'œuvre dans le hall était un acte global de conservation préventive. Ainsi protégée des intempéries et des cycles humidité-séchage, la Sphinge ne craignait plus la reprise de corrosion de ses armatures. La protection cathodique ne s'avérait donc plus nécessaire.

Pour autant, notre étude a permis pour la première fois d'évaluer les avantages et inconvénients de la protection cathodique. Il s'agit d'un traitement lourd, ayant des conséquences esthétiques et structurelles non négligeables du fait des percements qu'il implique. Il possède également une durée de vie limitée au-delà de laquelle les anodes doivent être changées. Cependant, le système peut être adapté, dans un esprit de collaboration avec les professionnels agréés, à chaque œuvre. Pour la conservation des sculptures d'extérieur, il constitue donc une voie de recherche prometteuse.

\section{Notes}

1. Mémoire encadré par M. Payre H. Boursier, E. Papet et E. Marie-Victoire : nous les remercions vivement.

2. Les analyses du matériau furent effectuées par le pôle béton du LRMH.

3. Nous tenons à les remercier tous les trois pour cette collaboration.

4. Le potentiel de corrosion se définit pour un milieu électrolytique donné. Cette grandeur décrit la tendance de chaque métal à se dissoudre dans ce milieu. L'échelle des potentiels est négative : plus E est grand, moins le métal a tendance à s'ioniser.

5. Substance conductrice permettant le passage des ions. Dans un béton, l'électrolyte est la solution interstitielle, c'est-à-dire l'eau circulant dans les pores du matériau.

6. Les anodes sont placées tous les x cm le long d'une armature, $x$ étant le rayon d'action d'une anode. Celui-ci est déterminé par la distance maximum parcourue par les ions issus des réactions galvaniques.
7. Un fragment de claustra provenant de la bourse de Toulouse et fourni par le LRMH fut utilisé pour les tests.

8. Aucune des anodes commercialisées ne possède le profil parfait pour être employée dans les sculptures : toutes doivent être modifiées.

9. Les anodes doivent obligatoirement être placées entre la surface du béton et l'armature. Elles ne peuvent pas être placées en dessous des fers ou même à l'intérieur d'une sculpture creuse.

\section{Bibliographie}

Audisio S., Béranger G., 2010, Anticorrosion et Durabilité dans le bâtiment, le génie civil et les ouvrages industriels, s.l., Presses polytechniques et universitaires romandes, Italie.

Marie-Victoire E., Bouichou M., 1996, Les altérations visibles du béton : définitions et aide au diagnostic, Champs-sur-Marne, Cercle des partenaires du patrimoine.
Raharinaïvo A., Arliguie G., Chaussadent T. et al., 1998, La corrosion et la protection des aciers dans le béton, Paris, Presses de l'Ecole nationale des Ponts et Chaussées, Collection du Laboratoire Central des Ponts et Chaussées.

Taché G. (dir.), 2012, Anodes galvaniques pour le traitement de la corrosion des armatures des constructions en béton, CEFRACOR Centre Français de l'Anticorrosion, commission Construction Bâtiment, Documents scientifiques et techniques, Paris.

\section{Document inédit}

Marec J.-C., 1996, Corrosion et protection cathodique, École d'application des techniques gazières, cours accompagnant la formation en protection cathodique. 